\title{
Thermal-Initiated Hydroxyethyl Methacrylate Functionalization of Multiwalled Carbon Nanotubes
}

\author{
Greg Curtzwiler, Philip J. Costanzo, Ray Fernando, Jeffrey E. Danes, Keith Vorst
}

ABSTRACT: Multiwalled-carbon nanotubes (MWCNTs) were functionalized via thermoinitiated free radical polymerization of 2-hydroxyethyl methacrylate (HEMA) using benzoyl peroxide. Tip sonication was used during the polymerization reaction to separate agglomerated nanotubes. The functionalization was confirmed by control experiments and attenuated total reflectance Fourier transform infrared spectroscopy (ATR-FTIR). Differential scanning calorimetry indicated that the addition of poly(HEMA)-MWCNTs to a two-component polyurethane coating will have little effect on the glass transition temperature of the coating. The poly-(HEMA)-functionalized MWCNTs formed large colloidal structures of highly dispersed nanotubes in both the non-sheared and sheared coatings as determined by atomic force microscopy. This study determined a quick and easy method to functionalize MWCNTs for incorporation into a two-component polyurethane coating. A simple method for producing ordered structures of the MWCNTs via shear observed.

\section{INTRODUCTION}

Previous studies have determined that carbon nano-tubes (CNTs) increase the mechanical properties of various systems. ${ }^{1-5}$ Various techniques have been used in an attempt to incorporate CNTs into engineering thermosets including polyurethanes and epoxies. ${ }^{1,6}$, Isolating nanotubes and creating favorable interactions between the nanotubes and the matrix will maximize mechanical increases in a composite. Sonication and chemical functionalization have been used to separate the agglomerates formed during synthesis creating more affinity for the matrix and surface area leading to a more stable system. ${ }^{6,8}$ Polymer functionalization of isolated nanotubes has proven to introduce sufficient steric repulsion to keep the CNTs isolated during processing. ${ }^{9}$ Gravity, viscosity, and interparticle forces must be considered when attempting to isolate and disperse CNTs into a medium as they are critical to stable systems.

Dispersion forces decrease in magnitude as $1 / D,{ }^{10}$ where $D$ is the distance between two molecules and is insufficient interaction with the medium to separate the nanotubes. The large surface area combined with insufficient attraction energy with the medium causes the CNTs to agglomerate hindering potential mechanical increases.

Sonication has been used to help overcome the strong dispersion forces of nanoparticles. ${ }^{8,9}$ When a medium contains nanoparticles, the mechanical and thermal properties are altered and the propagation of sound changes. Traveling sound waves through a medium induces particle motion, which creates a pressure wave. The pressure wave forces the nearby particles apart allowing for modifications to the system to increase system stability.

Stable suspensions of CNTs require the medium to adsorb to the surface of isolated nanotubes followed by a surface modification to avoid re-agglomeration. Polymeric materials have been widely used for nanoparticle stabilization. ${ }^{8-13}$ Additional stability is realized by steric repulsion of the particles as the free energy of the system increases from polymer overlap. The work required to concentrate the adsorbed polymer, as two particles interact, determines the stability of the suspension. The magnitude of repulsion is dependent on the polymer-solvent interaction and the thickness of the adsorbed layer. ${ }^{10} \mathrm{~A}$ good solvent allows for more configurations of the polymer and increases the hydrodynamic volume, which raises the entropy of the system; a bad solvent will cause the polymer chains to attract decreasing entropy. Concentrating the attached polymers introduces osmotic pressure and reduces the number of configurations for each polymer chain. 
Increased osmotic pressures decreases the entropy of the system, which is thermodynamically unfavorable resulting in particle separation. ${ }^{10,11}$ Thus, a longer polymer chain with a good solvent will produce better steric repulsion. In practice, the required thickness of the polymer layer is about an order of magnitude less than the radius of the particle. ${ }^{10}$

Adsorption (physisorption) is a nondestructive method to introduce steric stability as it relies only on van der Waals forces. Adsorption of polymers to a nanotube surface requires that a portion of the solvent be expelled from the solvated polymer and the surface where the polymer is to be adsorbed. ${ }^{9}$ The rate at which a polymer adsorbs is directly dependant on the particle-polymer and solvent-polymer interactions as well as molecular weight. ${ }^{9}$

Chemical modification is a common approach to add nanotube affinity toward a medium and retain nanotube isolation. ${ }^{1,5,6,11}$ Many methods have been developed to produce various functional groups on the side wall and caps of nanotubes. ${ }^{12}$ The functional groups created allow for subsequent reactions leading to increased affinity toward the host media. Polymer grafting has a similar effect as adsorption except the polymer chains are covalently bound to the nanotube wall.

The most common liquid-phase oxidations of CNTs consist of refluxing in nitric acid or ultrasonic treatment in a sulfuric/nitric acid mixture. The ultrasonic method yields shortened tubes covered with carboxyl groups, while the refluxing reaction is milder, reducing the degree of functionalization at the tube ends and defect sites. Oxidative attack at the defect sites opens the side wall creating functional groups such as phenols, quionines, lactones, carboxylic anhydrides, and acids. Much attention has been paid to reactions of amide and ester formations based on carboxylic chemistry. ${ }^{12}$

Surface initiated polymerization (SIP) allows for more control of the polymer functionalization. In this process, the initiating species must adsorb to the surface, create a highly reactive species that can propagate polymerization, and then react with monomer to commence the polymerization. ${ }^{10}$ SIP procedures have the advantage of minimally interfering with elaborate molecular framework that would compromise the nanotube's physical attributes. Wu et al. ${ }^{13}$ functionalized multiwalled carbon nanotubes (MWCNTs) with polystyrene via atom transfer radical polymerization, which yielded functionalities up to $50 \%$. The study suggests that CNTs can be activated by free radical initiators opening $n$-bonds for polymerization.

Various forces influence the CNT's location and motion once they are suspended in a liquid medium. Brownian motion distributes particles uniformly through dispersion forces while gravitational forces pull on the particle affecting the system's stability. The gravitational force on a particle suspended in a liquid is equal to the effective mass multiplied by the acceleration of gravity. The effective mass of a particle is the product of its volume and the density difference between the particle and the suspending liquid. ${ }^{9}$ When the gravitational force on a particle is substituted into the terminal velocity equation, there is a quadratic dependence on the radius for the sedimentation rate indicating the importance of particle size for system stability. The particles in solution eventually reach equilibrium where Brownian motion and gravitational sedimentation are balanced, resulting in a stable suspension. Thermal fluctuations, noise, and mechanical perturbations of the system as well as the size, density, and shape of the particle affect system equilibrium and can be tailored for more favorable interaction between the solvent and the particle. $^{9}$

The main objective of this study was to separate agglomerated MWCNTs via tip ultrasonication ${ }^{9,14}$ in a tetrahydrofuran (THF)/2-hydroxyethyl methacrylate (HEMA) solution for subsequent SIP of HEMA. The secondary objective was to determine if the one-dimensional alignment of nanoparticles observed in other studies could be reproduced using poly(HEMA)-MWCNTs in a two-component polyurethane coating. ${ }^{15-17}$ This was achieved by adding the poly(HEMA)-MWCNTs to a two-component polyurethane-coating formulation. The poly(HEMA)-MWCNT-poly-urethane coating was placed on a microscope slide, allowed to level by gravity, and cured. Two more coatings were made using a 37-pm 
drawdown cube to introduce shear to the system, cured, and then characterized by atomic force microscopy (AFM) to determine if nanoparticle alignment occurred on the surface of the coating. This study determined a quick, efficient method for functionalizing MWCNTs prepared for two-component polyurethane coatings, and reinforces the shear-induced one-dimensional alignment behavior of nanoparticles observed in previous studies.

\section{MATERIALS AND METHODS}

Materials

Unbundled, MWCNTs were used as received from Ahwahnee Technology (San Jose, CA). A modified acrylic copolymer polyol was used as received from BASF (Joncryl 910, Southfield, MI). Methyl 1,2,2,6,6- pentamethyl-4-piperidinyl ester (a hindered amine light stabilizer, Tinuvin 292) and poly(oxy-1,2-ethanediyl) (an ultraviolet light stabilizer, Tinuvin 1130) were used as received from Ciba Specialty Chemicals (Tarrytown, NY). A polyester-modified polymethylalkylsiloxane solution (a leveling additive, Byk 315) was used as received from Byk Chemie (Wallingford, CT). Hexamethyldiisocyanate trimer (Desmodur N3300A) was used as received from Bayer Material Science (Pittsburg, PA). All other items were received from commercially available sources and used without further purification.

\section{HEMA functionalization of MWCNT}

A $25-\mathrm{mL}$ round-bottomed flask was loaded with multiwalled carbon nanotube (MWCNT; 85 $\mathrm{mg})$, HEMA $(5 \mathrm{~mL}), \operatorname{THF}(5 \mathrm{~mL})$, and a magnetic stir bar and sealed with a rubber septum. A 0.25 in. diameter, tapered tip sonication horn was inserted through the septum until the tip of the horn was submerged in the liquid, ensuring an air tight seal. Benzoyl peroxide (BPO) (15 mg) was dissolved in THF $(0.5 \mathrm{~mL})$ and added to the reaction flask. The system was purged with nitrogen for $15 \mathrm{~min}$. The mixture was sonicated with a Misonix Ultrasonic Processor XL (Farmingdale, NY) equipped with a 0.25 -in. tapered horn for $1 \mathrm{~min}$ at $50 \mathrm{~W}$ and then placed in an oil bath at $80^{\circ} \mathrm{C}$. The mixture was removed from the oil bath and subjected to sonication for an additional $30 \mathrm{~s}$ (on the same power level) every $5 \mathrm{~min}$ until a total reaction time of $20 \mathrm{~min}$ was reached.

The highly viscous liquid was washed with a $50 \mathrm{vol} \%$ of THF/methanol three times then once more with 2-heptanone. A wash cycle consisted of sonicating at $50 \mathrm{~W}$ for $30 \mathrm{~s}$ in the washing solvent followed by $10 \mathrm{~min}$ of centrifugation at $4000 \mathrm{rpm}$. The supernatant was decanted off into a glass bottle, and the pellet was resuspended in fresh solvent via tip sonication at $50 \mathrm{~W}$ for $30 \mathrm{~s}$. The functionalized MWCNTs from the pellet were sonicated in 2-heptanone for $30 \mathrm{~s}$ at $50 \mathrm{~W}$ before addition to the coating formulation.

As a control experiment, a $25-\mathrm{mL}$ round-bottomed flask was loaded with HEMA (5 mL), THF (5 $\mathrm{mL})$, BPO $(10 \mathrm{mg})$, and a magnetic stir bar was then sealed with a rubber septum. The system was purged with nitrogen for $15 \mathrm{~min}$ and then placed in an oil bath at $80^{\circ} \mathrm{C}$ for $10 \mathrm{~min}$. Poly(HEMA) $(3 \mathrm{~g})$, confirmed by attenuated total reflectance Fourier transform infrared spectroscopy (ATR-FTIR), and MWCNTs $(100 \mathrm{mg}$ ) were added to methanol in a $50-\mathrm{mL}$ beaker and sonicated at $50 \mathrm{~W}$ for $1 \mathrm{~min}$. The system was left undisturbed for $24 \mathrm{~h}$ for observation. After observation, the MWCNTs were washed using the procedure described earlier.

Incorporation of HEMA-functionalized MWCNTs to a $2 \mathrm{~K}$ polyurethane coating

Part A of a two-component polyurethane automotive coating consisted of 58.7 wt \% Joncryl 910 acrylic polyol, 25.9 wt \% 2-heptanone, 8.11 wt \% hexanes, 5.90 wt \% n-pentyl propionate, 0.60 wt $\%$ Tinuvin 
292, 0.40 wt \% Tinuvin 1130, and 0.30 wt \% Byk 315 with 41.7 wt \% solids. The poly(HEMA) functionalized-multiwalled carbon nanotubes (MWCNTs) in 2-heptanone were added to the part A formulation for a $1 \mathrm{wt} \%$ concentration in the cured coating, mixed by hand for 2 min, and then conditioned at ambient temperature and pressure for $20 \mathrm{~min}$. Part B of the coating consisted of 54.5 wt \% Desmodur N3300A isocyanates and 45.5 wt \% n-butyl acetate with 54.4 wt \% solids.

Part $A$ was added to part $B$ in a $4: 1$ ratio, mixed by hand until there was a visually uniform viscosity then conditioned at ambient temperature and pressure for $20 \mathrm{~min}$. The mixture was placed on a glass slide via plastic transfer pipette, allowed to level by gravity, and then placed in an oven for $1 \mathrm{~h}$ at $70^{\circ} \mathrm{C}$. Two coatings were produced using a 37- $\mu \mathrm{m}$ draw-down cube at a moderate speed then placed in an oven for $1 \mathrm{~h}$ at $70^{\circ} \mathrm{C}$.

Attenuated total reflectance-Fourier transform infrared spectroscopy

Attenuated total reflectance-Fourier transform infrared spectroscopy (ATR-FTIR) was used to qualitatively determine the molecular composition in each composite. A Smart Performer ATR assembly (Thermo Scientific, Waltham, MA) attached to a Nexus 470 Fourier Transform Infrared Spectrometer (Nicolet Instruments, Offenbach, DE) scanned specimens at 32 scans per experiment. A background scan was performed before evaluation of all specimens. Each coating type was placed on the crystal at ambient temperature after the curing process. The washed poly(HEMA)-MWCNTs were placed in a glass jar and heated at $50^{\circ} \mathrm{C}$ in an oven until the solvent completely evaporated then placed on the ATR assembly and scanned. The pellet of the control experiment was scanned to ensure that the wash cycles were adequate to remove any poly(HEMA) that was not covalently attached to the MWCNTs. It should be noted that the pellet was scanned containing residual methanol to avoid inhalation hazards.

Differential scanning calorimetry

Poly(HEMA)-functionalized MWCNTs and each coating [poly(HEMA)-functionalized MWCNT/PU coating and PU coating] were placed in separate aluminum pans and hermetically sealed. The glass transition temperature of each coating and functionalized MWCNTs were evaluated on a TA Instruments Calorimeter model DSCQ1000 (TA Instruments, DE). The poly(HEMA)-MWCNT experiments consisted of a heat/cool/heat cycle between 20 and $150^{\circ} \mathrm{C}$ at a rate of $10^{\circ} \mathrm{C} / \mathrm{min}$ for the first heat cycle, $10^{\circ} \mathrm{C} / \mathrm{min}$ for the cooling cycle, and $20^{\circ} \mathrm{C} / \mathrm{min}$ for the second heat cycle in accordance with ASTM D3418-03 ${ }^{18}$ for the determination of the glass transition temperature. The experiments for the coatings had the same heating/cooling rates as the poly(HEMA)-MWCNTs, but were between -50 and $150^{\circ} \mathrm{C}$. Three specimens per coating type and poly(HEMA)-MWCNT were used.

\section{Atomic force microscopy}

The poly(HEMA)-functionalized MWCNT/PU films were analyzed with an optical microscope attached to a Pacific Nanotechnology AFM (Santa Clara, CA) to locate areas of interest. Areas of interest were scanned via AFM in contact mode using a NSC 36 type probe with a resolution of 256 lines/image and a scan angle of zero.

RESULTS

Poly(HEMA) functionalization of MWCNT 
The polymerization reaction was uniformly black and highly viscous, suggesting that the polymerization was successful. The supernatant after centrifugation of each wash was uniformly black indicating the presence of nanotubes with a strong affinity for the wash solvent. The CNTs were suspended in solution for over 1 month without disturbing the system.

After sonication of the control experiment, the system was allowed to stabilize under ambient conditions for $24 \mathrm{~h}$. The multiwalled carbon nanotubes (MWCNTs) were located at the bottom of the beaker indicating that the addition of poly(HEMA) to a MWCNT/methanol system will not increase the MWCNT's affinity for the solvent enough to keep them suspended in solution.

Attenuated total reflectance Fourier transform infrared spectroscopy

Three experiments were conducted on the poly (HEMA) and the washed MWCNTs from the control

experiment and the poly(HEMA)-MWCNTs [Fig. 1(a)] for the evaluation of molecular composition by ATR-

FTIR. The spectra of the poly(HEMA) from the control experiment and the poly(HEMA)MWCNTS

were compared against the Sprouse Polymer ATR library and had a correlation of 88 and $92 \%$, respec-

tively, with the reference spectra for poly-HEMA, indicating that polymerizations were successful. The

spectra of the MWCNT pellet from the control experiment [Fig. 1(b)] were also compared against

the reference library and did not have a correlation with poly-HEMA, and the MWCNT pellet spectra

did not have an absorbance at $1705 \mathrm{~cm}^{-1}$ (attributed to carbonyl stretching of the methacrylate group)

indicating that the centrifuge-washing technique was adequate to remove poly(HEMA) that was not cova-

lently attached to the MWCNTs [Fig. 1(b)]. The slight absorbance in the hydroxyl region can be attributed to residual methanol in the MWCNTs.

\section{Differential scanning calorimetry}

Three experiments were conducted on each coating type and the poly(HEMA)-functionalized MWCNTS

(Table I) for evaluation of the glass transition temperature at different rates of heating/cooling cycles. Statistical analysis using Tukey's 95\% simultaneous confidence interval (SAS, Cary, NC) indicated that the glass transition temperature for the poly(HEMA) functionalized MWCNT/PU coating was not statistically different from the PU coating under the test conditions of this study. This indicates that incorporating poly(HEMA)-functionalized MWCNT had little to no effect on the glass transition of the coating. The glass transition temperature for the poly(HEMA)- MWCNTs appears to be clearly higher than each coating under all conditions, but was not found to be statistically different according to the statistical model used in this study. This can be attributed to the high amount of variability in the poly(HEMA)-MWCNT results. The difference between the observed glass transition temperatures can be attributed to the various geometric configurations of the CNTs, which may introduce varying amounts of added steric hindrance altering the glass transition temperature.

Control experiments in conjunction with ATRFTIR and differential scanning calorimetry (DSC) indicated that MWCNTs can be functionalized via thermal initiated surface polymerization using 
relatively less hazardous materials and significantly faster than procedures described in the literature. ${ }^{12}$

For example, Nguyen et al. ${ }^{19}$ functionalized nonpurified MWCNTs with isocyanate functional groups by purifying the MWCNTs by refluxing in $9.5 \mathrm{M}$ nitric acid at $110^{\circ} \mathrm{C}$ for $24 \mathrm{~h}$ followed by heating with multifunctional isocyanates for an additional $7 \mathrm{~h}$. The total time of functionalization approached $48 \mathrm{~h}$ considering experimental setup, neutralization of the acid, and purification processes necessary compared to a functionalization time of $-1 \mathrm{~h}$ for MWCNTs prepared for similar systems as described in this study.

Poly(HEMA)-functionalized MWCNT/polyurethane composite coating

The optical clarity of the sheared coating was visually better than the nonsheared coating due to the thickness difference of the coatings. There were also fewer agglomerates that were observable without magnification when compared with the nonsheared coating. This may attributed to using a $37-\mu \mathrm{m}$ draw-down cube for application of the coating as it is possible that agglomerates larger than $37 \mu \mathrm{m}$ were removed from the coating due to clearance problems.

\section{Atomic force microscopy}

The sheared and nonsheared coatings were scanned with an AFM. The colloidal structures were located using the integrated optical microscope attached to the AFM then scanned with a resolution of 256 lines/image in contact mode with a scan angle of $0^{0}$. The scans indicated that the MWCNTs were well dispersed within the colloidal structures though agglomerates still present a problem (Fig. 2). The colloidal structures found in the coatings may be larger than $40 \mathrm{pm}$ in diameter (determined by optical microscopy, images not shown) though AFM scans do not show agglomerates larger than $15 \mathrm{pm}$ in diameter on the surface.

Two poly(HEMA)-functionalized MWCNT/polyurethane coatings were produced on glass slides with a single direction shear force (37-pm drawdown bar) to observe the effects of shear on the system. A particle suspended in a viscous liquid in motion will rapidly attain the velocity of the fluid in the same direction indicating that shear alignment of nanopar-ticles is plausible. ${ }^{9}$ When in motion, the velocity of the nanotubes would increase indefinitely except the increasing velocity of the particle simultaneously increase the viscous drag resulting in a negative component in the velocity vector slowing it down.${ }^{9}$ When the viscous drag of the particle equals the applied force, terminal velocity of the particle is achieved.

Scans of the colloidal structures in the sheared coating indicate that some of the CNTs were successfully isolated within the coating (Fig. 3). The even spacing of the CNTs exhibits the expected steric repulsion gained from polymer attachment.

Figure 3 shows the nanotubes aligned with the long axis normal to the shear direction with nearly equal spacing between the isolated tubes. This suggests nanolevel dispersion within the colloidal structures. The one-dimensional alignment of nanoparticles via shear has already been demonstrated with alumina and silica nanoparticles, ${ }^{15}$ but little has shown these effects for CNTs in a two-component polyurethane coating as reported here.

\section{CONCLUSIONS}


Unbundled, MWCNTs were functionalized via thermoinitiated free-radical polymerization of 2-HEMA with BPO. The functionalization was confirmed by ATR-FTIR and control experiments. DSC determined that the poly(HEMA)-MWCNT/PU coating was statistically the same as the PU coating for the glass transition temperature indicating that the introduction of poly(HEMA)-MWCNTs at 1 wt $\%$ has little effect on this property. The poly(HEMA)-functionalized MWCNTs formed large colloidal structures in both the nonsheared and sheared coatings as determined by optical microscopy indicating that the formulation of the coating should be modified. The colloidal structures do not appear to be agglomerates, but localized regions of highly dispersed MWCNTs as suggested by the AFM scans. The isolated tubes indicate that sonication can be used to successfully break apart most agglomerates, though some agglomerates remained in the coating that were $-15 \mu \mathrm{m}$ in diameter. The viscous drag created by the applied shear force aligned the MWCNTs with the long axis normal to the shear direction indicating that shear alignment is possible in this system. To confirm the behavior observed by AFM, the study should be reproduced, and other characterization tools should be used such as transmission electron microscopy to ensure that this behavior is not only found on the surface of the coating. The macroscopic effects of the addition of functionalized nanotubes on a two-component polyurethane coating should also be determined using characterization techniques such as dynamic mechanical analysis, thermogravimetric analysis, electromechanical tensile testing, scratch resistance, chemical resistance, and accelerated weathering. This study determined a quick and easy method to functionalize MWCNTs for incorporation into a twocomponent polyurethane coating and reinforces the shear-induced one-dimensional alignment behavior of nanoparticles observed in previous studies.

\section{References}

1. Aglan, H.; Dennig, P.; Ganguli, S.; Irvin, G. J Reinf Plast Compos 2006, 25, 175.

2. Chen, P.; He, J.; Hu, G.-H.; Zhang, B.; Zhang, J.; Zhang, Z. Carbon 2006, 44, 692.

3. Esawi, A. M. K.; Farag, M. M. Mater Des 2007, 28, 2394.

4. Chen, W.; Tao, X.; Liu, Y. Compos Sci Technol 2006, 66, 3029.

5. Yaping, Z.; Aibo, Z.; Qinghua, C.; Jiaoxia, Z.; Rongchang, N. Mater Sci Eng 2006, 435, 145.

6. Curtzwiler, G.; Singh, J.; Miltz, J.; Doi, J.; Vorst, K. J Appl Polym Sci 2008, 109, 218.

7. Nguyen, T.; Granier, A.; Pellegrin, B.; Celina, M. Proceedings of the Nanotech 2008 Conference. Boston, MA, June 1-5, 2008.

8. Wang, X.; Xiong, J.; Yang, X.; Zheng, Z.; Zhou, D. Polymer 2006, 47, 1763.

9. Morrison, I.; Ross, R. Colloidal Dispersion: Suspension, Emulsion, and Foams; Wiley: New York, NY, 2002.

10. Butt, H. J.; Graf, K.; Kappl, M. Physics and Chemistry of Interfaces; Wiley-VCH Verlag: Berlin, 2006.

11. Florian, H.; Jacek, N.; Zbigniew, R.; Karkl, S. Chem Phys Lett 2003, 370, 820.

12. Burghard, M. Surf Sci Rep 2005, 58, 1.

13. Wu, H.; Tong, R.; Qiu, X.; Yang, H.; Lin, Y.; Cai, R.; Qian, S. Carbon 2007, 45, 152.

14. Bittmann, B.; Haupert, F.; Schlarb, A. Ultrason Sonochem 2009, 16, 622.

15. Brickweg, L.; Floryancic, B.; Sapper, E.; Fernando, R. J Coat Technol Res 2007, 4, 107.

16. Cohen, I.; Mason, T.; Weitz, D. Chem Phys Lett 2004, 93, 1.

17. Xue, W.; Grest, G. Chem Phys Lett 1990, 64, 419.

18. ASTM D3418-03. Standard Test Method for Transition Temperatures and Enthalpies of Fusion and Crystallization of Polymers by Differential Scanning Calorimetry. ASTM International, West Conshohocken, PA.

19. Nguyen, T.; Granier, A.; Steffens, K.; Lee, H.-J.; Martin, J. Retrieved from: http://www.nist.gov/customcf/get_pdf.cfm? pub_id1/4903766. 


\section{Top $\uparrow$}

(a)

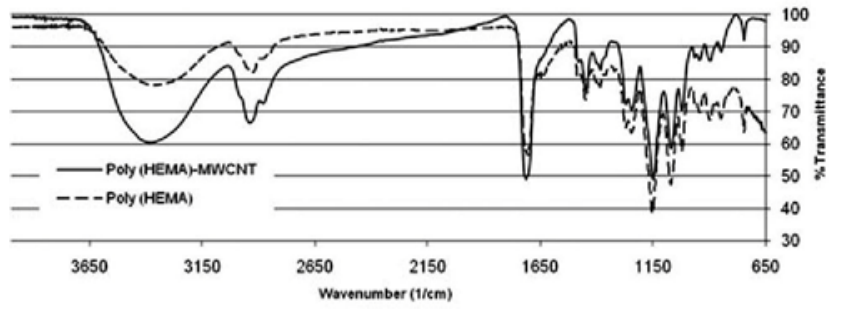

(b)

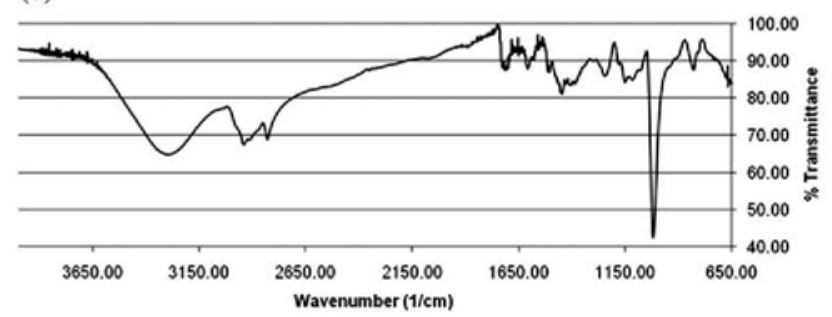

Figure 1 Attenuated total reflectance Fourier transform infrared spectroscopy of poly(HEMA) functionalized MWCNT (A), poly(HEMA) (a), and nonfunctionalized MWCNT after the washing cycles (b).

TABLE I

Glass Transition Temperatures ( $\left.T_{g}\right)$ of Poly(HEMA)-

MWCNT, PU Coating, and Poly(HEMA-MWCNT/PU

Coating $^{a}$

\begin{tabular}{|c|c|c|c|}
\hline $\mathrm{T}_{\mathrm{g}} 10 \mathrm{C} / \mathrm{mi}$ & $10 \mathrm{C} / \mathrm{mir}$ & $0 \mathrm{C} / \mathrm{min}$ & \\
\hline Sample & Heat $\left({ }^{\circ} \mathrm{C}\right)$ & $\mathrm{Cool}\left({ }^{\circ} \mathrm{C}\right)$ & Heat $\left({ }^{\circ} \mathrm{C}\right)$ \\
\hline $\begin{array}{l}\text { Poly(HEMA)- } \\
\text { MWCNT }\end{array}$ & $48.23 a$ & $39.43 \mathrm{a}$ & $62.19 \mathrm{a}$ \\
\hline Poly(HEMA & CNT/ & & \\
\hline PU coating & $35.60 a b$ & $29.13 \mathrm{a}$ & $40.66 \mathrm{~b}$ \\
\hline $\begin{array}{l}\text { PU coating } \\
\text { P value* }\end{array}$ & $\begin{array}{c}37.77 \mathrm{ab} \\
0.024\end{array}$ & $\begin{array}{c}28.47 \mathrm{a} \\
0.080\end{array}$ & $\begin{array}{c}42.06 \mathrm{~b} \\
0.004\end{array}$ \\
\hline
\end{tabular}

a Samples with the same letter in a column were not found to be significantly different using Tukey's $95 \%$ simultaneous confidence interval.

* $\mathrm{P}$ values represent differences between each heat, cool, and heat cycle column. 


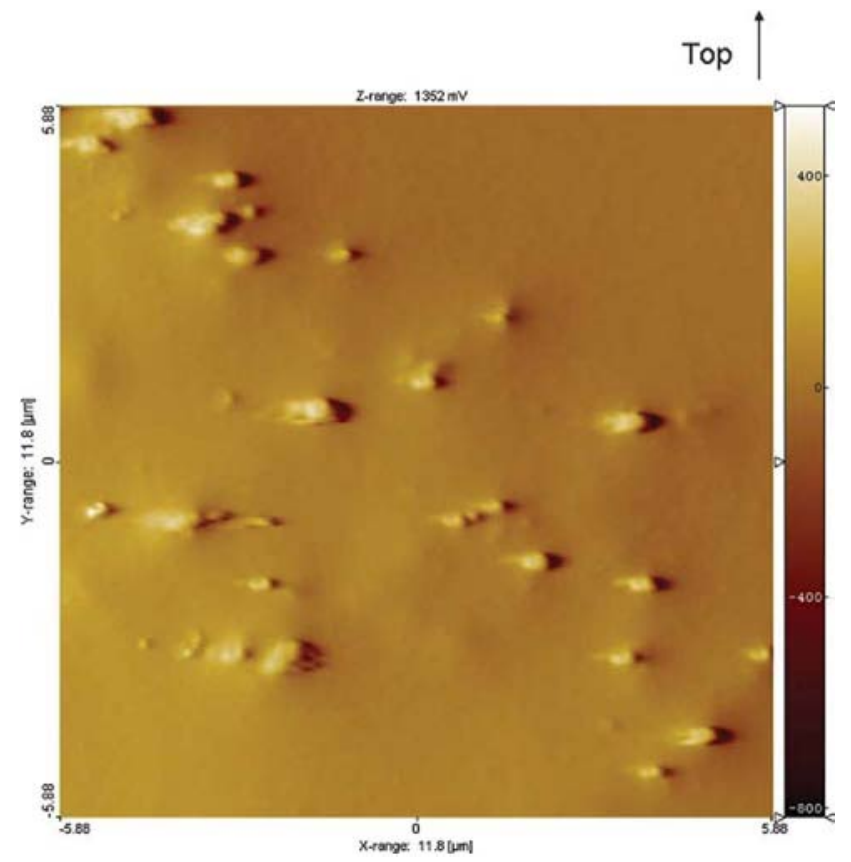

Figure 2 Functionalized carbon nanotubes with poly(HEMA) in a two-component polyurethane coating without shear. The image is $-12 \times 12 \mu \mathrm{m}$. [Color figure can be viewed in the online issue, which is available at wileyonlinelibrary. com. ]

$$
\text { Top } \uparrow
$$

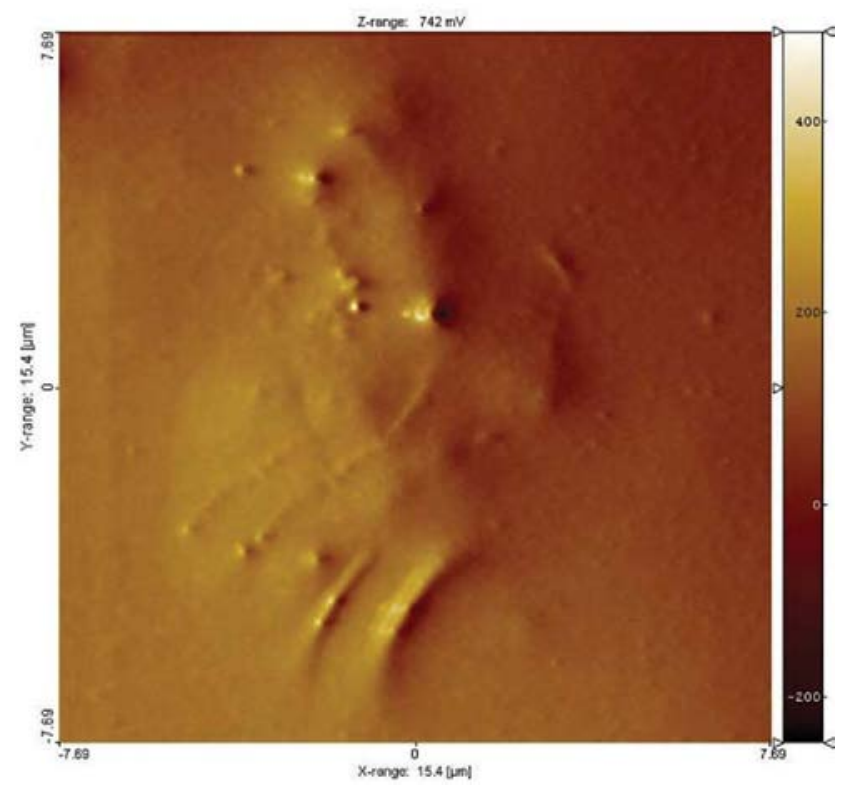

Figure 3 The sheared poly(HEMA)-functionalized multi-wall carbon nanotubes are isolated and evenly spaced with the long axis aligned normal to the direction of shear (left to right). The image is $-15 \times 15 \mu \mathrm{m}$. [Color figure can be viewed in the online issue, which is available at wileyonlinelibrary. com. ] 